\title{
DISTRIBUCIÓN GEOGRÁFICA Y COLABORACIÓN INTERNACIONAL DE LAS PUBLICACIONES CIENTIIFICAS LATINOAMERICANAS Y DEL CARIBE SOBRE TUBERCULOSIS EN PUBMED
}

\author{
Cristina Torres-Pascual(101,2,a, Héctor Javier Sánchez-Pérez(13,4,b, Pilar Ávila-Castells@55,6,c \\ 1 Escola Universitària de la Salut i l’Esport, Universitat de Girona, Girona, España. \\ 2 Red GRAAL España. \\ 3 El Colegio de la Frontera Sur, San Cristóbal de Las Casas, México. \\ ${ }^{4}$ Red GRAAL México. \\ 5 Facultad de Enfermería, Universitat de Girona. \\ 6 Servei de Medicina Interna. Parc Hospitalari Martí i Julià, Girona, España. \\ Licenciada en Documentación, doctora en Información y Documentación; ${ }^{\mathrm{b}}$ médico, doctor en Medicina; ${ }^{\mathrm{c}}$ licenciada en \\ Medicina y Cirugía, doctora en Medicina y Cirugía.
}

\section{RESUMEN}

Objetivo: Describir la cobertura geográfica de las publicaciones científicas de América Latina y el Caribe sobre tuberculosis y su colaboración internacional. Materiales y métodos: Los registros fueron recuperados de la base de datos bibliográfica PubMed (2009-2018), limitando la búsqueda a países latinoamericanos y del Caribe. Los artículos se analizaron en varias categorías, como número total, países participantes, instituciones, autores, colaboraciones y citas. Se utilizó el software UCINET y VOSviewer para representar las redes de colaboración. Resultados: Se recuperaron 2495 artículos, 4,2\% de la producción mundial. Más de la mitad de las investigaciones proceden de Brasil (52,5\%), México (16,6\%) y Perú (10,5\%). El 97,6\% de la producción presenta coautoría, con una tasa de colaboración internacional del $24,5 \%$. Los países con mayor producción en colaboración internacional son Brasil (39,1\%), Perú (26,8\%) y México (16\%), además son los que presentan mejor centralidad, cercanía e intermediación. Estados Unidos (11,9\%), Reino Unido (5,8\%), Francia (2,1\%), España y Suiza (1,7\%), Sudáfrica (1,7\%) e Italia (1,6\%), son los principales colaboradores con Latinoamérica. Conclusión: Brasil, junto a Perú y México, es líder de la producción científica sobre tuberculosis en Latinoamérica y el Caribe, por el volumen de artículos y su posición en la red de colaboración. La erradicación de la tuberculosis pasa por continuar investigando en colaboración internacional, y así llevar a cabo políticas de salud más efectivas en tuberculosis.

Palabras clave: Tuberculosis; Indicador de Colaboración; Bibliometría; Red Social; América Latina; Región del Caribe (fuente: DeCS BIREME).

\section{GEOGRAPHICAL DISTRIBUTION AND INTERNATIONAL COLLABORATION OF LATIN AMERICAN AND CARIBBEAN SCIENTIFIC PUBLICATIONS ON TUBERCULOSIS IN PUBMED}

Citar como: Torres-Pascual C, SánchezPérez HJ, Àvila-Castells P. Distribución geográfica y colaboración internacional de las publicaciones científicas latinoamericanas y del Caribe sobre tuberculosis en PubMed. Rev Peru Med Exp Salud Publica. 2021;38(1):4957. doi: https://doi.org/10.17843/ rpmesp.2021.381.5726.

Correspondencia: Cristina TorresPascual; Avda. Francesc Macià 65, 17190 Salt, Girona. España; ctorres@euses.cat

Recibido: 05/05/2020 Aprobado: 25/11/2020 En línea: 01/03/2021

\section{ABSTRACT}

Objective: The objective of the study was to describe the geographical coverage of Latin American and Caribbean scientific publications on tuberculosis and its international collaboration. Materials and methods: The records were retrieved from the PubMed database (2009-2018), limiting the search to Latin American and Caribbean countries. Articles were analyzed in several categories, such as the total number, countries, institutions, authors, collaborations, and citations. The software UCINET and VOSviewer were used. Results: 2,495 items were recovered, $4.2 \%$ of world production. More than half of the research came from Brazil (52.5\%), Mexico (16.6\%) and Peru (10.5\%). 97.6\% of the production is co-authored, with a rate of international collaboration of $24.5 \%$. The countries with the highest production in international collaboration are Brazil (39.1\%), Peru (26.8\%) and Mexico (16\%); they are also the countries with the best OutDegree, OutCloseness and Betweenness. The United States (11.9\%), United Kingdom (5.8\%), France (2.1\%), Spain and Switzerland (1.7\%), South Africa (1.7\%) and Italy (1.6\%) are the main collaborators with Latin America. Conclusion: Brazil, together with Peru and Mexico, is the undisputed leader in Latin American and Caribbean tuberculosis production, due to the volume of articles and its position in the collaborative network. The eradication of tuberculosis requires continuing research in international collaboration, to carry out more effective health policies on tuberculosis.

Keywords: Tuberculosis; Bibliometrics; Social Networking; Latin America; Caribbean Region (Source: MeSH NLM). 


\section{INTRODUCCIÓN}

La tuberculosis (TB) es la primera causa de muerte por enfermedad infecciosa y se encuentra entre las diez primeras causas de mortalidad a nivel mundial ${ }^{(1)}$. Pese a que su incidencia está descendiendo, todavía se está lejos de alcanzar los Objetivos de Desarrollo Sostenible para erradicarla en el $2030^{(2)}$. La estrategia impulsada por la Organización Mundial de la Salud (OMS) de «Fin a la TB» pretende disminuir la incidencia de la enfermedad en un $90 \%{ }^{(3)} \mathrm{y}$ su mortalidad en un $95 \%$ entre 2015 y $2035^{(4)}$.

Ante la prevalencia de TB, su retraso en la detección clínica y, por consiguiente, en su diagnóstico y tratamiento, la aparición de cepas multirresistentes, así como la afectación de poblaciones especialmente vulnerables, la OMS ha puesto énfasis en la importancia de intensificar los estudios sobre TB como una medida prioritaria para su erradicación ${ }^{(5)}$. Para ello se requiere de estudios llevados a cabo en colaboración internacional ${ }^{(6)}$. Establecer redes de cooperación facilita la gestión y optimización de recursos, permite compartir infraestructuras y capacidades, hecho especialmente importante en países en desarrollo. Asimismo, posibilita generar y distribuir conocimiento a nivel interdisciplinar y aumentar la productividad de los estudios, favoreciendo la innovación en salud ${ }^{(7)}$. Así, el uso de análisis de redes sociales para evaluar las pautas de colaboración se convierte en una herramienta esencial para proporcionar información y establecer nuevas políticas de investigación. El análisis de redes sociales permite observar y describir las relaciones entre distintos elementos bibliográficos, como autores o países, proporcionando una estructura visible y organizada de flujos de información.

Si bien existen distintos estudios bibliométricos que analizan el comportamiento de las publicaciones sobre TB a nivel internacional ${ }^{\left({ }^{(8)}\right.}$, en áreas geográficas concretas, como Korea ${ }^{(9)}$ o Brasil ${ }^{(10)}$, o sobre la colaboración internacional de la producción latinoamericana en TB de Brasil, Perú, México, Colombia y Argentina ${ }^{(11)}$; no se ha identificado ningún estudio bibliométrico centrado en contribución internacional de todos los países latinoamericanos y del Caribe.

PubMed es la base de datos internacional más utilizada en biomedicina por las características y volumen de trabajos indizados, por su antigüedad y funcionalidad a nivel mundial ${ }^{(12)}$. Su acceso gratuito, versus otras bases de datos, como Scopus, hace que sea la base de datos más consultada. Si bien LILACS (Literatura Latinoamericana y del Caribe en Ciencias de la Salud) y SciELO (Scientific Electronic Library Online) presentan mayor cantidad de revistas latinoamericanas indizadas, estas están publicadas mayoritariamente en español y portugués. Este hecho a priori refleja una menor internacionalización de las investigaciones, puesto que países como Estados Unidos de América (EUA) o Reino Unido, en caso de colaborar con países latinoamericanos, prefieren revistas anglosajonas para que las investigaciones tengan mayor difusión.

\section{MENSAJES CLAVE}

Motivación para realizar el estudio: Dado el alto impacto de la tuberculosis en Latinoamérica y el Caribe, se realizó un estudio bibliométrico para identificar los países de Latinoamérica y el Caribe que realizan más investigaciones en tuberculosis, e identificar la red de colaboración de éstos con el resto del mundo.

Principales hallazgos: Más de la mitad de las investigaciones proceden de Brasil. Uno de cada cuatro artículos se realizó con colaboración internacional. Estados Unidos, Reino Unido, y Francia son los principales colaboradores con Latinoamérica.

Implicancias: La erradicación de la tuberculosis debe apoyarse en la colaboración internacional que permita diseñar políticas de salud más efectivas.

El objetivo del este estudio es describir la cobertura geográfica de las publicaciones científicas de América Latina y el Caribe sobre TB y su colaboración internacional en PubMed.

\section{MATERIAL Y MÉTODOS}

\section{Diseño del estudio}

Estudio descriptivo retrospectivo de enfoque bibliométrico.

\section{Recuperación y tratamiento de los datos}

La recuperación de registros se obtuvo el 22 de diciembre de 2019, de la base de datos de acceso libre PubMed por ser una de las bases de datos internacionales más importante en el campo biomédico que sirve de referencia para los profesionales e investigadores de la salud en la región latinoamericana. La consulta de las citaciones recibidas se hizo en Web of Science. Los términos de búsqueda fueron tuberculosis o TB (título, abstract o MeSH) refinados para países latinoamericanos y del Caribe, que se muestran en la siguiente estrategia de búsqueda, para el periodo 2009-2018: (tuberculosis[Title/Abstract] OR TB[Title/Abstract] OR tuberculosis[MeSH] OR TB[MeSH]) AND (Argentina[Affiliation] OR Belice[Affiliation] OR Brasil[Affiliation] OR Brazil[Affiliation] OR Bolivia[Affiliation] OR Chile[Affiliation] OR Colombia[Affiliation] OR Costa Rica[Affiliation] OR Cuba[Affiliation] OR El Salvador[Affiliation] OR Ecuador[Affiliation] OR Haiti[Affiliation] OR Honduras[Affiliation] OR Guatemala[Affiliation] OR Jamaica[Affiliation] OR Mexico[Affiliation] OR Panama[Affiliation] OR Paraguay[Affiliation] OR Peru[Affiliation] OR Puerto Rico[Affiliation] OR Dominican Republic[Affiliation] OR Republica Dominicana[Affiliation] OR Uruguay[Affiliation] OR Venezuela[Affiliation]) Filters: from 2009 - 2018. Se recuperaron 4205 registros. 
El filtrado de datos se basó en la eliminación de registros incompletos, registros atribuidos por error a determinados países, como es el caso de Nuevo México, o que no desarrollaron el tema de la TB. De ello resultaron 2495 registros y posteriormente se normalizaron los campos de país, autores e instituciones de afiliación.

\section{Análisis de los indicadores bibliométricos}

Los indicadores bibliométricos analizados fueron: a) Producción científica total (número y porcentaje de artículos indizados) y porcentaje de la producción latinoamericana a partir del total de registros indizados en PubMed; b) Tasa de crecimiento para medir la evolución porcentual de la producción (2009-2018); c) Relación entre el número acumulado anual de artículos y la cobertura temporal por las ecuaciones lineales, logísticas y exponenciales; d) Instituciones latinoamericanas y del Caribe con diez o más trabajos y autores más prolíficos ( $\geq 10$ artículos) ${ }^{(13)}$ y su afiliación, según constó en las autorías en que los autores firman en primera posición. En caso de que el autor no tuviera producción como primer firmante se tuvo en cuenta la autoría como último firmante; e) Nivel colaborativo para medir la profundidad de la colaboración (relación entre número total de autores o países y el número total de artículos) y tasa de colaboración internacional para observar la amplitud (porcentaje de artículos firmados por dos o más países); f) Correlación entre el número de artículos y número de artículos en colaboración por país con la correlación de Pearson; g) Número de citaciones total por país y promedio de citas recibidas con su desviación estándar (DE). El número de citas utilizadas fue la que directamente ofreció Web of Science en cada registro; h) La estructura de la red social de colaboración internacional se analizó a través de la densidad de la red, centralidad de grado y su valor normalizado, centralidad de cercanía y centralidad de intermediación y su valor normalizado ${ }^{(14)}$.

La densidad de la red oscila entre 0 (red dispersa) y uno (fuertemente conectada). La centralidad de grado es el número de vínculos de un nodo (autor, país) en la red, por ello, a mayor conexión del nodo mayor prestigio tiene dentro de la red. La centralidad de cercanía es la distancia entre nodos de la red, establece que, a mayor cercanía, la capacidad de interacción con otros miembros es mejor. La centralidad de intermediación es el número de caminos más cortos que pasan a través de un nodo dado, señala que a mayor intermediación, mayor capacidad de flujo de la información transmitida entre los otros nodos.

\section{Análisis de datos}

Los registros de PubMed se exportaron a Refworks y posteriormente a Excel para el manejo de los datos. Una vez obtenidos los países que cooperan entre sí, se realizó la tabla con la matriz correspondiente en excel para su exportación a UCINET $6.0^{(14)}$. Las redes se visualizaron con NetDraw, integrado en el paquete UCINET. Posteriormente se exportó a VOSviewer (Visualization of Similarities viewer) ${ }^{(15)}$ para visualizar la red social de colaboración internacional. La representación gráfica de los resultados se obtuvo a partir del algoritmo de clústering de VOSviewer. Para las coautorías se emplearon los valores default de VOSviewer. La imagen que ofrece VOSviewer identifica la cantidad de artículos producidos por el tamaño de los nodos y la intensidad de relación que conecta los distintos nodos por el grosor de las líneas de enlace. Los nodos que corresponden al mismo clúster se reconocen por el color. Para el análisis estadístico se utilizó Microsoft Excel ${ }^{\circledR}$. Los parámetros estadísticos utilizados fueron: frecuencias absolutas, porcentajes, media y desviación estándar (DE) y correlación de Pearson.

\section{Consideraciones éticas}

Al tratarse de un estudio basado en datos de artículos científicos, no se consideró ser sometido para la aprobación de un comité de ética.

\section{RESULTADOS}

La producción sobre TB indizada en PubMed (2009-2018) procedente de países latinoamericanos y del Caribe es de 2495 artículos, 4,2\% de la producción mundial. A lo largo del periodo se observa un crecimiento exponencial $\left(\mathrm{R}^{2}=0,9\right)$ (Figura 1), con una tasa de crecimiento del 133,9\%. En los últimos cuatro años se acumula el 50\% de la producción. Un total de 611 artículos están publicados de forma colaborativa con dos o más países. En este caso el crecimiento no atiende a una tendencia exponencial sino lineal $\left(\mathrm{R}^{2}=0,8\right)$ (Figura 1$)$. En la relación entre el número acumulado anual de artículos y la cobertura temporal, las ecuaciones lineales obtuvieron un alto coeficiente de determinación $(0,9)$, mientras que para la exponencial y logística fue de 0,9 y 0,8 , respectivamente, en tanto que, para la producción en colaboración internacional, solo se ajusta el crecimiento lineal y logarítmica, con un coeficiente de determinación de 0,8 y 0,5 , respectivamente (Figura 1).

La producción incluye un total de 10594 autores de 7383 instituciones pertenecientes a 22 países latinoamericanos y 70 del resto del mundo. El 52,5\% de los artículos procede de Brasil, seguido de México (16,6\%) y Perú (10,5\%). De ellos, los países con un mejor ajuste en el crecimiento exponencial de la literatura, por acercarse a un $\mathrm{R}^{2}$ de 1, son Perú y Brasil. El 97,6\% de la producción presenta coautoría, con una tasa de colaboración internacional del 24,5\%. El nivel de colaboración entre autores fue de 7,6 (DE 4,4), entre instituciones de 5,01 (DE 2,3) y, entre países de 3 (DE 1,8). Brasil es el país con más producción en colaboración internacional $(39,1 \%)$ seguido de Perú $(26,8 \%)$ y México (16\%) (Tabla 1). 


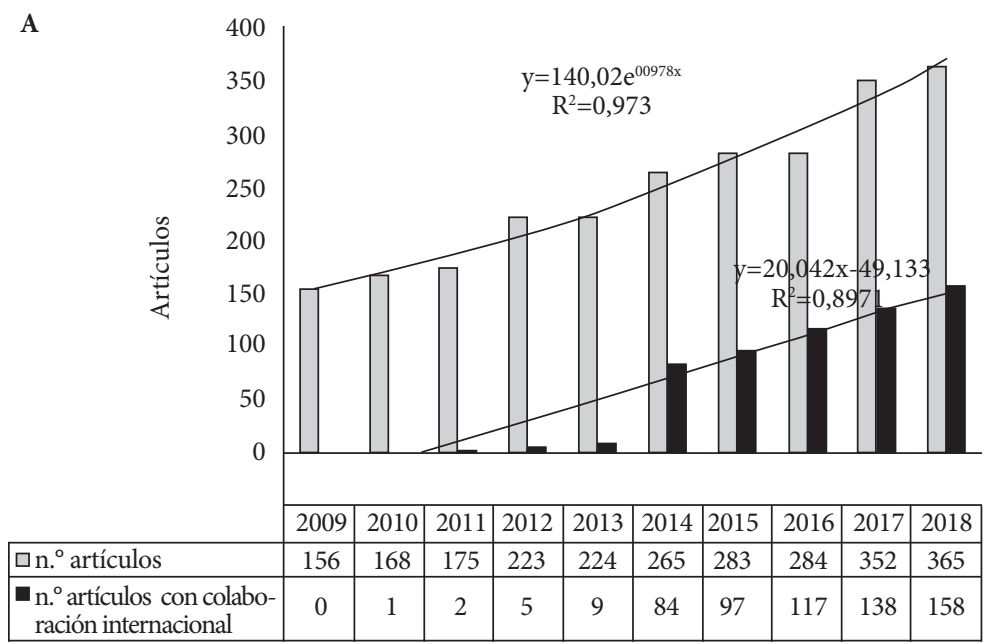

B

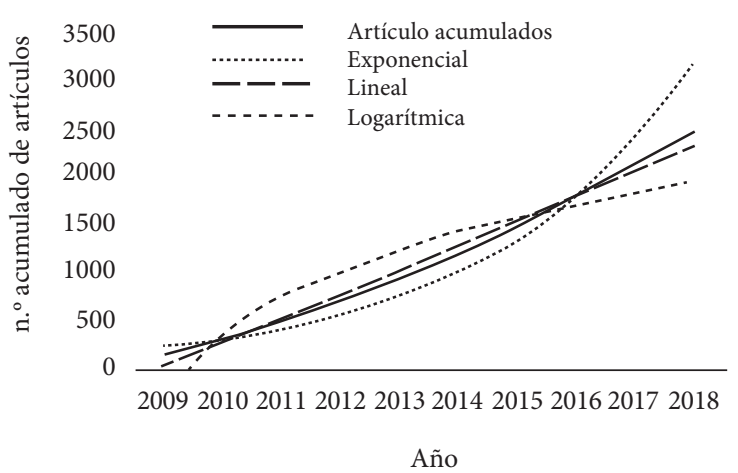

C

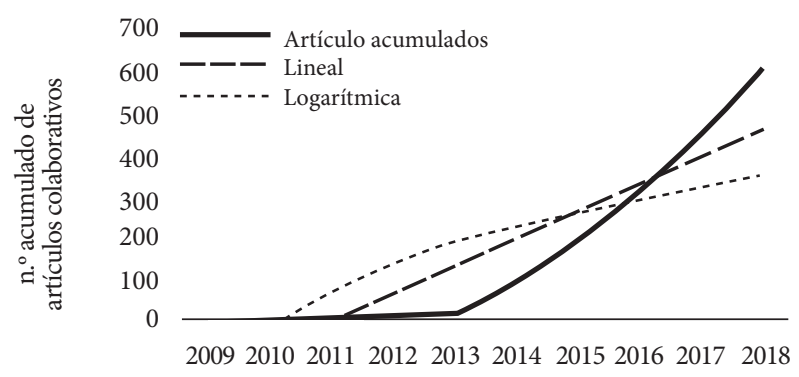

Año

Figura 1. A. Evolución del número de artículos y producción colaborativa. B. Relación entre el número acumulado de artículos y año. C. Relación entre el número acumulado de artículos colaborativos y año de publicación.

La producción analizada presenta una correlación de 0,9 entre países con mayor producción y países con mayor número de trabajos en colaboración internacional. La producción suma un total de 23635 citaciones. La media de citaciones de la producción sin colaboración internacional se sitúa en el 8,5 (DE 16,6), mientras que para la producción internacional es de 10,9 (DE 25,8). En la mayoría de casos aparece una DE más alta que el promedio, debido a la alta dispersión de las citaciones.

Las diez instituciones con mayor producción en colaboración internacional son: Universidad Peruana Cayetano Heredia (47,1\%), Socios en Salud (15\%) y la Asociacion Benefica PRISMA (14\%) de Perú; Fundacao Oswaldo Cruz de Brasil (12,6\%); Instituto de Medicina Tropical Alexander von Humboldt, de Perú (7,4\%); Instituto Nacional de Ciencias Médicas y Nutrición «Salvador Zubirán» de México (6,9\%); University of Sao Paulo de Brasil (5,9\%); Instituto Mexicano del Seguro Social (IMSS) (5,2\%); Universidade Federal do Rio de Janeiro (4,7\%); y, Universidade Federal do Rio Grande do Sul (4,6\%) de Brasil.

Los autores, más prolíficos ( $\geq 10$ artículos), que trabajan en colaboración internacional son: R.H. Gilman $(\mathrm{n}=61)$ de la
Universidad Peruana Cayetano Heredia, Asociación Benéfica PRISMA de Perú y Johns Hopkins Bloomberg School of Public Health de EUA; D. Moore $(n=43)$ de la Universidad Peruana Cayetano Heredia de Perú y London School of Hygiene and Tropical Medicine de Reino Unido; C.A. Evans $(\mathrm{n}=37)$ de la Asociación Benéfica PRISMA y Universidad Peruana Cayetano Heredia de Perú e Imperial College London and Wellcome Trust Imperial College Centre for Global Health Research de Reino Unido; C. Seas ( $n=26)$ del Instituto de Medicina Tropical Alexander von Humboldt, Universidad Peruana Cayetano Heredia y Hospital Nacional Cayetano Heredia de Perú; L. Lecca ( $\mathrm{n}=30)$ de Socios en Salud y Ministerio de Salud de Perú, y Harvard Medical School y Brigham and Women's Hospital de EUA; R. Calderón $(n=15)$ de Socios en Salud Perú y Universidade Federal do Rio de Janeiro de Brasil; J. Galea $(n=12)$ de Socios en Salud Perú y Harvard Medical School de EUA; J. Grandjean ( $n=16)$ de Socios en Salud Perú e Institute of Child Health University College London de Reino Unido; R. Hernández-Pando ( $\mathrm{n}=55$ ) del Instituto Nacional de Ciencias Médicas y Nutrición «Salvador Zubirán» de México; A. Trajman $(n=42)$ de la Universidade Federal do Rio de Ja- 
Tabla 1. Cobertura geográfica de la producción científica de Latinoamérica y el Caribe sobre TB indizada en PubMed (2009-2018).

\begin{tabular}{|c|c|c|c|c|c|c|}
\hline País & $\begin{array}{l}\text { Artículos } \\
\text { n (\%) }\end{array}$ & $\begin{array}{c}\text { Citaciones } \\
\text { n }\end{array}$ & $\begin{array}{c}\text { Artículos sin } \\
\text { colaboración } \\
\text { internacional } \\
\mathbf{n}(\%)\end{array}$ & $\begin{array}{c}\text { Promedio } \\
\text { citaciones sin } \\
\text { colaboración } \\
\text { internacional } \\
\text { (DE) }\end{array}$ & $\begin{array}{l}\text { Artículos cola- } \\
\text { boración inter- } \\
\text { nacional } \mathbf{n}(\%)\end{array}$ & $\begin{array}{l}\text { Promedio citaciones } \\
\text { colaboración inter- } \\
\text { nacional (DE) }\end{array}$ \\
\hline Brasil & $1310(52,5)$ & 11192 & $1071(42,9)$ & $8,0(16,1)$ & $239(39,1)$ & $11,6(25,2)$ \\
\hline México & $414(16,6)$ & 4342 & $316(12,7)$ & $10,1(17,7)$ & $98(16,0)$ & $12,1(23,7)$ \\
\hline Perú & $261(10,5)$ & 2442 & $97(3,9)$ & $8,0(11,1)$ & $164(26,8)$ & $10,2(16,3)$ \\
\hline Colombia & $189(7,6)$ & 1163 & $143(5,7)$ & $6,3(11,1)$ & $46(7,5)$ & $5,7(10,3)$ \\
\hline Argentina & $184(7,4)$ & 2584 & $128(5,9)$ & $14,1(26,6)$ & $56(9,2)$ & $13,9(34,7)$ \\
\hline Cuba & $44(1,8)$ & 539 & $33(1,3)$ & $5,0(7,8)$ & $11(1,8)$ & $34,3(134,0)$, \\
\hline Venezuela & $34(1,34)$ & 307 & $22(0,9)$ & $10,4(13,1)$ & $12(2,0)$, & $6,8(6,2)$ \\
\hline Chile & $51(2,0)$ & 234 & $36(1,4)$ & $3,1(3,9)$ & $15(2,4)$ & $8,7(9,8)$ \\
\hline Uruguay & $20(0,8)$ & 218 & $10(0,4)$ & $15(21,8)$ & $10(1,6)$ & $6,8(6,6)$ \\
\hline Haití & $27(1,1)$ & 217 & $5(0,2)$ & $3,6(4,3)$ & $22(3,6)$ & $12,0(14,9)$ \\
\hline Ecuador & $16(0,6)$ & 92 & $7(0,3)$ & $3,9(4,9)$ & $9(1,5)$ & $7,22(10,1)$ \\
\hline Jamaica & $16(0,6)$ & 88 & $2(0,1)$ & 0 & $14(2,3)$ & $6,3(5,2)$ \\
\hline Panamá & $12(0,5)$ & 57 & $4(0,2)$ & $9,5(11,4)$ & $8(1,3)$ & $2,4(3,7)$ \\
\hline Honduras & $7(0,3)$ & 40 & $3(0,1)$ & $9,8(5,5)$ & $4(0,6)$ & $0,3(0,6)$ \\
\hline Puerto Rico & $2(0,1)$ & 40 & $2(0,1)$ & $20(28,3)$ & 0 & 0 \\
\hline Paraguay & $5(0,2)$ & 28 & 0 & 0 & $5(0,8)$ & $5,6(6,8)$ \\
\hline Bolivia & $5(0,2)$ & 22 & 0 & 0 & $5(0,8)$ & $4,4(7,6)$ \\
\hline Guatemala & $5(0,2)$ & 21 & $1(0,0)$ & 0 & $4(0,6)$ & $4,2(5,8)$ \\
\hline Nicaragua & $1(0,0)$ & 9 & 0 & 0 & 0 & 0 \\
\hline Costa Rica & $1(0,0)$ & 0 & 0 & 0 & $1(0,2)$ & 0 \\
\hline El Salvador & $5(0,2)$ & 0 & $1(0,0)$ & 0 & $4(0,6)$ & 0 \\
\hline $\begin{array}{l}\text { República Domi- } \\
\text { nicana }\end{array}$ & $1(0,0)$ & 0 & $1(0,0)$ & 0 & 0 & 0 \\
\hline
\end{tabular}

neiro de Brasil y McGill University Health Centre de Canada; A. Kritski ( $n=67)$ de la Rede Brasileira de Pesquisas em Tuberculose (REDE-TB) y de Universidade Federal do Rio de Janeiro de Brasil; B.B. Andrade ( $\mathrm{n}=15)$ de Fundação Oswaldo Cruz, Universidade Salvador, Escola Bahiana de Medicina e Saúde Pública de Basil y National Institutes of Health de EUA; y; R. Dietze $(n=26)$ de Universidade Federal do Espírito Santo de Brasil (Figura 2). En todos ellos, parte de su producción la firman como primer o último autor.

Los países latinoamericanos y del Caribe en las investigaciones sobre TB cooperan con 70 países. De éstos destacan los EUA (11,9\%); Reino Unido (5,8\%); Francia $(2,1 \%)$ y España y Suiza (1,7\% cada uno) (Tabla 2). El 25,1\% de los trabajos en colaboración internacional se establece únicamente entre los mismos países latinoamericanos, con un nivel de colaboración de 2,2 (DE 0,5). En la Figura 3 se aprecia la relación entre países que investigan de forma conjunta.

La red analizada presenta una densidad de 0,6. EUA es el país con mayor porcentaje de vínculos con países latinoamericanos (6,3\%), seguido de Brasil (5,4\%), Reino Unido $(3,7 \%)$ y Perú $(3,7 \%)$. Los países con mayor porcentaje de intermediación son Brasil (15,4\%), EUA (11,9\%), Reino Unido $(8,8 \%)$ y Francia $(5,7 \%)$. Los países con mayor cercanía son Brasil $(79,8)$, EUA $(78,4)$ y Reino Unido $(74,6)$ (Tabla 3).

\section{DISCUSIÓN}

La TB, tal como muestra el incremento de investigaciones en los últimos años, tanto a nivel mundial como en países latinoamericanos y del Caribe ${ }^{(16)}$, suscita interés entre los 


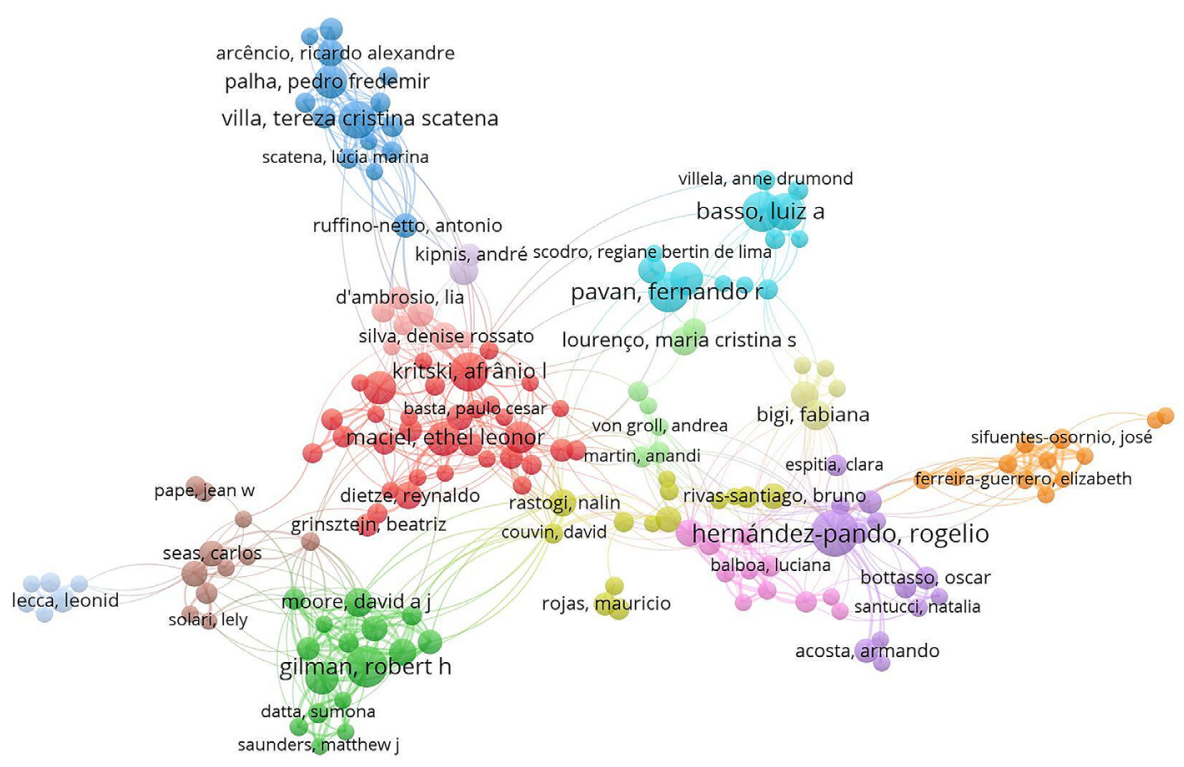

Figura 2. Red internacional de colaboración de autores con diez o más artículos.

investigadores y tomadores de decisiones. Aunque los resultados del estudio presentan un incremento de la actividad científica a lo largo del tiempo, se aprecian grandes variaciones entre países. En general, los países de Latinoamérica más productivos en investigación en salud son Brasil, México y Argentina, y los de mayor impacto y colaboración son Perú, Puerto Rico y Argentina. En el campo de la TB este comportamiento es ligeramente diferente ${ }^{(17)}$, donde Brasil

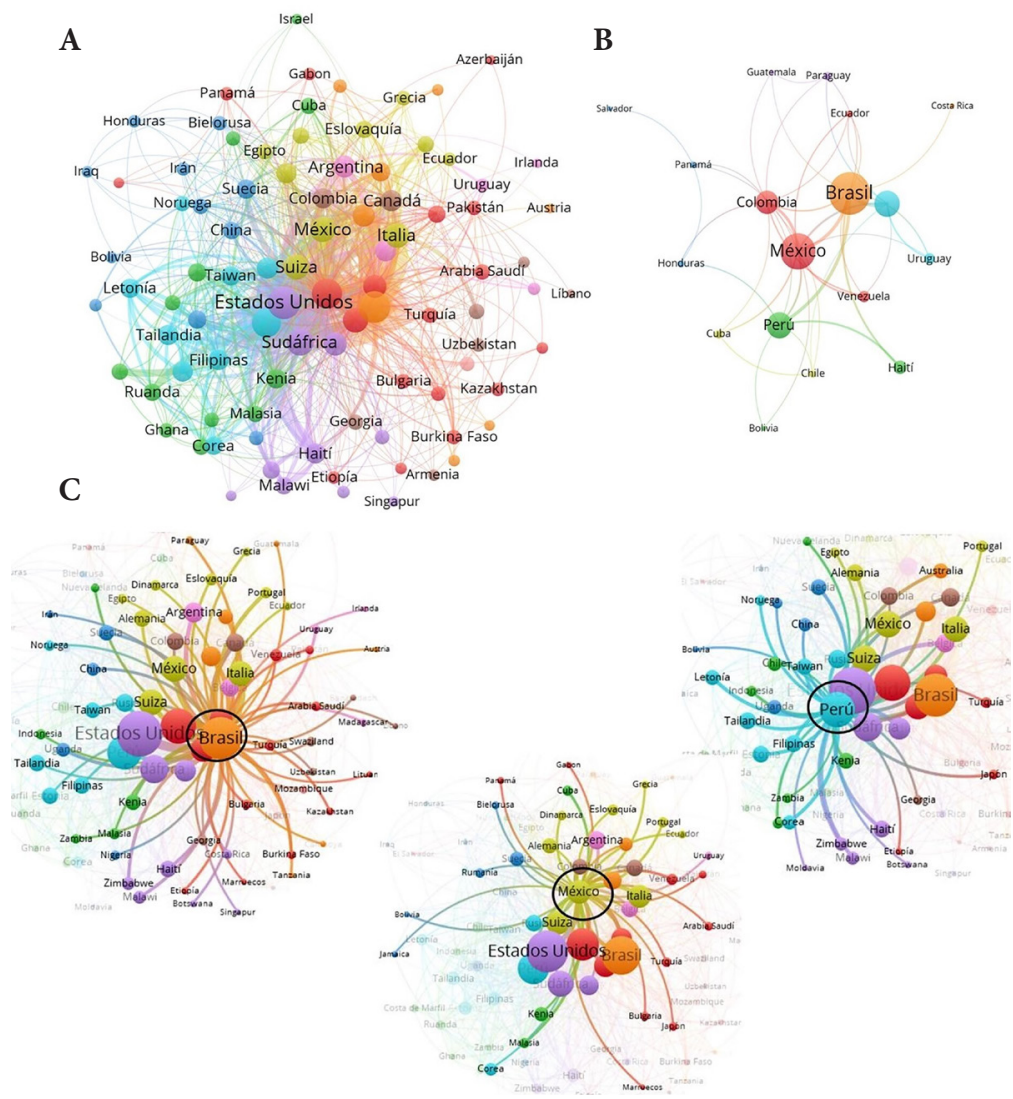

Figura 3. A. Representación de la red de colaboración internacional de los países de América Latina y el Caribe. B. Colaboración entre los países de América Latina y el Caribe. C. Colaboración aislada de Brasil, México y Perú. 
Tabla 2. Cobertura geográfica de los países colaboradores de la producción de Latinoamérica y el Caribe sobre TB indizada en PubMed (2009-2018).

\begin{tabular}{|c|c|c|c|c|c|}
\hline País & $\begin{array}{l}\text { Artículos } \\
\text { n (\%) }\end{array}$ & País & $\begin{array}{c}\text { Artículos } \\
\text { n (\%) }\end{array}$ & País & $\begin{array}{c}\text { Artículos } \\
\text { n (\%) }\end{array}$ \\
\hline Estado Unidos & $298(11,9)$ & Letonia & $7(0,3)$ & Madagascar & $3(0,1)$ \\
\hline Reino Unido & $145(5,8)$ & Mozambique & $7(0,3)$ & Ruanda & $3(0,1)$ \\
\hline Francia & $52(2,1)$ & Malawi & $6(0,2)$ & Zimbabue & $3(0,1)$ \\
\hline España & $43(1,7)$ & Arabia Saudí & $5(0,2)$ & Bielorrusia & $2(0,1)$ \\
\hline Suiza & $43(1,7)$ & Dinamarca & $5(0,2)$ & Botsuana & $2(0,1)$ \\
\hline Sudáfrica & $42(1,7)$ & Indonesia & $5(0,2)$ & Costa de Marfil & $2(0,1)$ \\
\hline Italia & $39(1,6)$ & Japón & $5(0,2)$ & Gabón & $2(0,1)$ \\
\hline Canadá & $35(1,4)$ & Nigeria & $5(0,2)$ & Irlanda & $2(0,1)$ \\
\hline India & $31(1,2)$ & Noruega & $5(0,2)$ & Kazakstán & $2(0,1)$ \\
\hline Bélgica & $27(1,1)$ & Pakistán & $5(0,2)$ & Singapur & $2(0,1)$ \\
\hline Suecia & $24(1,0)$ & Eslovaquia & $5(0,2)$ & Uzbekistán & $2(0,1)$ \\
\hline Australia & $21(0,8)$ & Burkina Faso & $4(0,2)$ & Zambia & $2(0)$, \\
\hline Alemania & $19(0,8)$ & Georgia & $4(0,2)$ & Armenia & $1(0,0)$ \\
\hline Países Bajos & $19(0,8)$ & Nueva Zelanda & $4(0,2)$ & Azerbaiyán & $1(0,0)$ \\
\hline China & $16(0,6)$ & Romania & $4(0,2)$ & Camboya & $1(0,0$ \\
\hline Portugal & $16(0,6)$ & Tanzania & $4(0,2)$ & Ghana & $1(0,0)$ \\
\hline Rusia & $12(0,5)$ & Turquía & $4(0,2)$ & Iraq & $1(0,0)$ \\
\hline Uganda & $11(0,4)$ & Vietnam & $4(0,2)$ & Israel & $1(0,0)$ \\
\hline Tailandia & $10(0,4)$ & Bulgaria & $3(0,1)$ & Kenia & $1(0,0)$ \\
\hline Malasia & $9(0,4)$ & Egipto & $3(0,1)$ & Líbano & $1(0,0)$ \\
\hline Estonia & $8(0,3)$ & Etiopía & $3(0,1)$ & Marruecos & $1(0,0)$ \\
\hline Filipinas & $8(0,3)$ & Grecia & $3(0,1)$ & Moldavia & $1(0,0)$ \\
\hline Corea & $7(0,3)$ & Irán & $3(0,1)$ & Ucrania & $1(0,0)$ \\
\hline
\end{tabular}

se posiciona como el país con más vínculos de cooperación, y Perú el que posee instituciones y autores más prolíficos. Brasil, Perú y México, países latinoamericanos con más del $50 \%$ de casos estimados de TB en Latinoamérica ${ }^{(18)}$, son los más prolíficos en estudios de campo, Brasil y México ocupan la sexta y decimoctava posición, respectivamente, en la producción mundial sobre salud ${ }^{(19)}$.

Brasil destaca por ser el país con mayor producción sobre TB ${ }^{(7)}$, e incrementar los trabajos científicos en colaboración ${ }^{(10)}$. Aunque Haití tiene un elevado número de casos estimados de TB, su presencia en la producción analizada es muy escasa. A pesar de ello, su tendencia a trabajar en colaboración con EUA, favorece dicha producción ${ }^{(19)}$. El interés creciente de Brasil, Perú y, en menor medida de México, junto a los países con altos ingresos que colaboran con éstos, refleja su compromiso científico sobre el campo en estudio ${ }^{(8)}$. EUA, al formar parte de la región Americana de la OMS, ha establecido vínculos con Latinoamérica y el Caribe, lo que si bien permitió lograr ciertos Objetivos de Desarrollo del Milenio en 2015, en el caso de la TB las metas establecidas no se alcanzaron ${ }^{(20)}$.

Brasil, centro de la red de investigación de América Latina, ha incrementado el número de investigaciones en cola- boración con Argentina, Chile y México ${ }^{(21)}$. Esta situación se hace extensible a las investigaciones sobre TB a excepción de Perú, tercer país con mayor número de colaboraciones con Brasil. El que Brasil participe en distintas redes mundiales estructuradas sobre TB como la Brazilian Rede TB o Global TB Network GTN $^{(22)}$ favorece su potencialidad para trabajar de forma conjunta ${ }^{(23)}$.

En 2015 se establecieron planes nacionales de investigación en TB en varios países de carga alta y media de TB, como Brasil, Rusia, India, China y Sudáfrica, países del denominado BRICS. Estos países concentran casi el 50\% de la carga mundial de $\mathrm{TB}{ }^{(24)}$, y se han convertido en líderes académicos del Programa Nacional de Tuberculosis BRICS, participando en la Red de Investigación de TB BRICS. Los resultados obtenidos muestran una fuerte colaboración entre Sudáfrica e India, país prolífico en este campo de estudio, pero no sucede lo mismo con el resto de países BRICS. La tendencia de estos países es colaborar con países con altos ingresos y no entre ellos ${ }^{(16)}$. La distribución geográfica de la cooperación entre países de Latinoamérica y el Caribe se da en mayor medida entre países geográficamente próximos ${ }^{(25)}$, y puede decirse que su producción en investigación es, 
Tabla 3. Parámetros de centralidad en la red de colaboración internacional de la producción de Latinoamérica y el Caribe sobre TB indizada en PubMed (2009-2018).

\begin{tabular}{|c|c|c|c|}
\hline País & $\begin{array}{c}\text { Grado } \\
\text { (grado normalizado) }\end{array}$ & Cercanía & $\begin{array}{c}\text { Intermediación } \\
\text { (intermediación } \\
\text { normalizada) }\end{array}$ \\
\hline Brasil & $488(5,4)$ & 79,8 & $1259,2(15,0)$ \\
\hline Perú & $377(3,7)$ & 66,4 & $227,2(2,8)$ \\
\hline México & $175(1,9)$ & 64,1 & $202,7(2,5)$ \\
\hline Argentina & $105(1,2)$ & 61,1 & $258,8(3,2)$ \\
\hline Colombia & $74(0,8)$ & 60,7 & $149,3(1,8)$ \\
\hline Haití & $67(0,8)$ & 53,8 & $8,4(0,1)$ \\
\hline Chile & $47(0,8)$ & 56,2 & $29,9(0,4)$ \\
\hline Cuba & $28(0,3)$ & 53,5 & $4,4(0,0)$ \\
\hline Venezuela & $33(0,4)$ & 55,1 & $15,8(0,2)$ \\
\hline Jamaica & $16(0,2)$ & 47,9 & $0,6(0,0)$ \\
\hline Ecuador & $14(0,1)$ & 52,9 & $4,6(0,0)$ \\
\hline Uruguay & $13(0,1)$ & 51,1 & 0 \\
\hline Panamá & $10(0,1)$ & 50 & $14,5(0,2)$ \\
\hline Honduras & $10(0,1)$ & 45,3 & $9,1(0,1)$ \\
\hline Paraguay & $8(0,1)$ & 50,8 & $2,6(0,0)$ \\
\hline Guatemala & $7(0,1)$ & 49,7 & $0,2(0,0)$ \\
\hline Bolivia & $6(0,1)$ & 49,2 & 0 \\
\hline Costa Rica & $6(0,1)$ & 47,4 & $1,1(0,0)$ \\
\hline El Salvador & $4(0,0)$ & 48,4 & 0,5 \\
\hline
\end{tabular}

en general, escasa. Ello podría deberse a la baja proporción del producto bruto interno que estos países dedican a la investigación.

A nivel mundial, países con menor incidencia de TB como Australia, EUA, Italia, Noruega, Países Bajos y Reino Unido, llevan a cabo políticas de contribución transfronteriza ${ }^{(26)}$. Los flujos migratorios conducen a países como España ${ }^{(27)} \mathrm{o}$ Reino Unido ${ }^{(26)}$ a realizar estudios sobre $\mathrm{TB}^{(27)}$, preferentemente en colaboración con aquellos países con larga trayectoria en el control de la TB. Las características migratorias de la frontera de México con EUA favorecen la transmisión de enfermedades infecciosas como la TB y contribuyen a la colaboración de ambas naciones para su control.

La mayoría de países pueden obtener y compartir información rápidamente, dado que no existen grandes diferencias en los valores de centralidad de cercanía ${ }^{(7)}$. La ubicación privilegiada en la red de colaboración de Brasil, Perú y México, hace que tengan un alcance más fácil y rápido para acceder a recursos y otros miembros de la red, hecho que contribuye a establecer nuevas colaboraciones. Éstos al presentar un mayor grado de cercanía y menos intermediarios respecto a los demás países latinoamericanos, están en una posición de tener información más rápida que el resto. Igualmente, Brasil, Perú y México ocupan posiciones de conexión entre grupos que les permite trabajar con interfaces entre subgrupos de actores fuertemente unidos y controlar el flujo de información entre éstos. Perú, pese a tener menor volumen de trabajos que
México, tiene un mayor comportamiento en la red, muy probablemente debido a una alta incidencia de TB multifármaco-resistente ${ }^{(28)}$.

EUA, país que más invierte en investigaciones sobre $\mathrm{TB}^{(16)} \mathrm{y}$ el Reino Unido, son los líderes indiscutibles de la producción en colaboración con países emergentes principalmente con Brasil, México, Perú y Argentina. La centralidad de grado de estos cuatro países refleja su compromiso para investigar sobre la TB, así como su interés en trabajar conjuntamente con países en desarrollo ${ }^{(7)}$. Ello favorece a que los artículos en colaboración con éstos tengan una mayor visibilidad y reciban mayor número de $\operatorname{citas}^{(21)}$.

Pese a que las investigaciones en colaboración no llegan al $25 \%$, el valor favorable de la densidad la red, al ser superior al $50 \%{ }^{(29)}$, y la centralización asociada con la gran cantidad de países que participan, sugiere un buen patrón de cohesión en la colaboración en los estudios sobre TB. Estos resultados destacan la necesidad de continuar en esta dirección, poniendo mayor atención en dar apoyo a aquellos países que precisan de mayores oportunidades en el fortalecimiento de nuevas colaboraciones. La internacionalización del conocimiento favorece las posibilidades de fomentar el trabajo conjunto entre los investigadores ${ }^{(29)} \mathrm{y}$, así, las investigaciones podrán contribuir al desarrollo de métodos de prevención, diagnóstico y tratamiento más efectivos ${ }^{(30)}$.

El uso de PubMed permitió mostrar la baja presencia de autores e instituciones de Latinoamérica y el Caribe en la literatura sobre TB en una de las bases de datos bibliográficas de acceso libre más consultadas. A diferencia de bases de datos como LILACS o SciELO, PubMed indexa prácticamente la totalidad de las revistas en inglés, lo que favorece que las investigaciones llevadas a cabo por autores latinoamericanos tengan una mayor visibilidad a nivel mundial. No obstante, los investigadores de países más pequeños tienen menos probabilidad de publicar en revistas anglosajonas, por lo que publican en revistas indexadas en bases de datos regionales. En tal sentido, el uso de una sola base de datos supone una limitación y sería deseable ampliar en futuros estudios la consulta a Scopus, LILACS o SciELO. Otra limitación es la falta de representación de todos los países latinoamericanos en Web of Science, lo que supone un sesgo en el sistema de análisis de citas. Además, para conocer algún sesgo de publicación sería recomendable también analizar las fuentes de financiamiento, externa y nacional, pública y privada. Finalmente, el estudio no reflejaría la totalidad de instituciones, puesto que PubMed entre 2009 y 2013 no indicaba todas las afiliaciones de los autores y colaboradores.

En conclusión, a lo largo de la última década se ha visto un aumento de las publicaciones sobre TB. Brasil, junto a Perú y México, es líder indiscutible de la producción sobre TB en Latinoamérica y el Caribe, al ser el país con mayor número de artículos y su posición en la red de co- 
laboración. La erradicación de la TB pasa por incrementar aún más el número de investigaciones, preferentemente con colaboración internacional, para poder establecer políticas de salud más efectivas y optimizar recursos.

Contribuciones de los autores: CT, PA y HJ han participado en la concepción y diseño del artículo. CT en la recolección de resultados y análisis e interpretación de datos. CT, PA y HJ han participado en la redacción del artículo, revisión crítica del artículo, aprobación de la versión final y asumen responsabilidad frente a los contenidos del manuscrito.

Financiamiento: Autofinanciado.

Conflictos de interés: Los autores no tienen ningún conflicto de interés que declarar.

\section{REFERENCIAS BIBLIOGRÁFICAS}

1. World Health Organization. Global tuberculosis report 2019. [Internet]. World Health Organization; 2019 [consultado el 27 de marzo del 2020]. Disponible en https://www.who.int/tb/publications/global_report/en/.

2. Abajobir AA, Abate KH, Abbafati C, Abbas KM, Abd-Allah F, Abdulkader $\mathrm{RS}$, et al. Measuring progress and projecting attainment on the basis of past trends of the health-related Sustainable Development Goals in 188 countries: an analysis from the Global Burden of Disease Study 2016. Lancet. 2017;390(10100):1423-459. doi:10.1016/s0140-6736(17)32336-x.

3. World Health Organization. The End TB Strategy. [Internet]. World Health Organization; 2015 [consultado el 27 de marzo del 2020]. Disponible en: https://www.who.int/tb/strategy/es/.

4. Kritski A, Dalcolmo MP, Mello FCQ, Carvalho ACC, Silva DR, Oliveira MM, et al. The role of the Brazilian Tuberculosis Research Network in national and international efforts to eliminate tuberculosis. J Bras Pneumol. 2018;44(2):77-81. doi: 10.1590/S1806-37562017000000435.

5. World Health Organization. Global strategy and targets for tuberculosis prevention, care and control after 2015 [Internet]. World Health Organization; 2015 [consultado el 27 de marzo del 2020]. Disponible en: https:// www.who.int/tb/post_2015_tb_presentation.pdf.

6. Fonseca e Fonseca B, Pereira da Silva MV, Mendonça de Araújo K, Barros Sampaio R, Ozório Moraes M. Network analysis for science and technology management: Evidence from tuberculosis research in Fiocruz, Brazil. PLoS One. 2017;12(8):e0181870. doi: 10.1371/journal.pone.0181870.

7. Fonseca e Fonseca B, Barros Sampaio R, de Araújo Fonseca MV, Zicker F Co-authorship network analysis in health research: method and potential use. Health Res Policy Syst. 2016;14:34. doi: 10.1186/s12961-016-0104-5.

8. Nafade V, Nash M, Huddart S, Pande T, Gebreselassie N, Lienhardt C, et al. A bibliometric analysis of tuberculosis research, 2007-2016. PLoS One. 2018;13(6): e0199706. doi: 10.1371/journal.pone.0199706.

9. Lee HW, Yim JJ. A Bibliometric Analysis on Tuberculosis Research of Korea from 1979 to 2017. J Korean Med Sci. 2019;34(11):e95. doi:10.334 6/jkms.2019.34.e95.

10. Guimarães Vasconcellos A, Medicis Morel C. Enabling Policy Planning and Innovation Management through Patent Information and $\mathrm{Co}-\mathrm{Au}-$ thorship Network Analyses: A Study of Tuberculosis in Brazil. PLoS One. 2012;7(10):e45569. doi: 10.1371/journal.pone.0045569.

11. Migliori GB, Centis R, D’Ambrosio L, Silva DR, Rendon A. International collaboration among medical societies is an effective way to boost Latin American production of articles on tuberculosis. J Bras Pneumol. 2019;45(2):e20180420. doi: 10.1590/1806-3713/ 20180420.

12. Trueba-Gómez R, Estrada-Lorenzo JM. La base de datos PubMed y la búsqueda de información científica. Semin Fund Esp Reumatol. 2010;11(2):49-63.

13. Ramos JM, Masiá M, Padilla S, García-Pachón E, Gutiérrez F. Producción española sobre tuberculosis a través del MEDLINE (1997-2006). Arch Bronconeumol. 2009;45(6):271-278. doi: 10.1016/j.arbres.2008.09.011.

14. Petrescu-Prahova M, Belza B, Leith K, Allen P, Coe NB, Anderson LA. Using Social Network Analysis to Assess Mentorship and Collaboration in a Public Health Network. Prev Chronic Dis. 2015;12:150103. doi: 10.5888/ pcd12.150103.
15. Wang Y, Zheng J, Zhang A, Zhou W, Dong H. Visualization maps for the evolution of research hotspots in the field of regional health information networks. Inform Health Soc Care. 2018;43(2):186-206. doi: 10.1080/17538157.2017.1297304.

16. Organización Panamericana de la Salud. Tuberculosis en las Américas 2018. Washington, D.C.: OPS; 2018.

17. Zacca-González G, Chinchilla-Rodríguez Z, Vargas-Quesada B. Medical scientific output and specialization in Latin American countries. Scientometrics. 2018;115(3):1635-165

18. Muñoz del Carpio-Toia A, Sánchez-Pérez HJ, Verges de López C, López-Dávila LM, Sotomayor-Saavedra MA, Sorokin P. Tuberculosis en América Latina y el Caribe: reflexiones desde la bioética. Pers Bioét. 2018;22(2):331-357. doi: 10.5294/pebi.2018.22.2.10.

19. Yao Q, Chen K, Yao L, Lyu P, Yang T, Luo F, et al. Scientometric trends and knowledge maps of global health systems research. Health Res Policy Syst. 2014;12:26. doi: 10.1186/1478-4505-12-26.

20. United Nations. Millenium Declaration. General Assembly, Fifty-fifth session of the General Assembly of the United Nations; 2000 Sep 5 to 2001 Sep 11; New York (NY), US. New York, UN, 2000 (Document A/ RES/55/2). www.un.org/millennium/declaration/ares552e.pdf.

21. Adams J. The rise of research networks. Nature. 2012;490:335-336. doi:10.1038/490335a.

22. Silva DR, Rendon A, Alffenaar JW, Chakaya JM, Sotgiu G, Esposito S, et al. Global TB Network: working together to eliminate tuberculosis. J Bras Pneumol. 2018;44(5):347-349. doi: 10.1590/S1806-37562018000000279.

23. Sotgiu G, Sulis G, Matteelli A. Tuberculosis-a World Health Organization Perspective. Microbiol Spectr. 2017;5(1). doi: 10.1128/microbiolspec. TNMI7-0036-2016.

24. Raviglione M, Uplekar M, Weil D, Kasaeva T. Tuberculosis makes it onto the international political agenda for health...finally. Lancet Glob Health. 2018;6(1):e20-e21. doi: 10.1016/S2214-109X(17)30449-7.

25. Su Y, Long C, Yu Q, Zhang J, Wu D, Duan Z. Global scientific collaboration in COPD research. Int J Chron Obstruct Pulmon Dis. 2017;12:215-225. doi: 10.2147/COPD.S124051.

26. Dara M, Sulis G, Centis R, D’Ambrosio L, de Vries G, Douglas P, et al. Cross-border collaboration for improved tuberculosis prevention and care: policies, tools and experiences. Int J Tuberc Lung Dis. 2017;21(7):727-736. doi: 10.5588/ijtld.16.0940.

27. Sánchez-Montalvá A, Salvador F, Molina-Morant D, Molina I. Tuberculosis e inmigración. Enferm Infecc Microbiol Clin. 2018;36(7):446-455. doi: 10.1016/j.eimc.2017.10.006.

28. Quispe N, Asencios L, Obregon C, Velásquez GE, Mitnick CD, Lindeborg $\mathrm{M}$, et al. The fourth national anti-tuberculosis drug resistance survey in Peru. Int J Tuberc Lung Dis. 2020;24(2):207-213. doi: 10.5588/ijtld.19.0186.

29. Gesell SB, Barkin SL, Valente TW. Social network diagnostics: a tool for monitoring group interventions. Implement Sci. 2013;8:116. doi: 10.1186/1748-5908-8-116.

30. Aria-Guillén M, Escalante P, Palacios Gutiérrez JJ. Nuevas perspectivas en infección tuberculosa latente. Arch Bronconeumol. 2020;56(2):74-75. doi: 10.1016/ j.arbres.2019.07.009. 